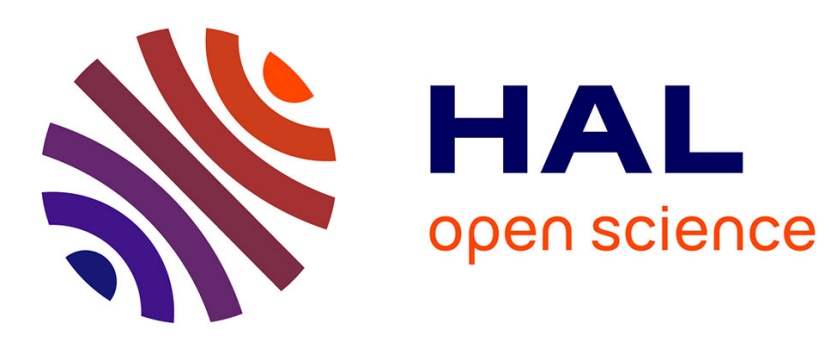

\title{
Développements techniques en matière d'accélérateurs au Laboratoire de l'Accélérateur Linéaire d'Orsay
}

B. Mouton

\section{To cite this version:}

B. Mouton. Développements techniques en matière d'accélérateurs au Laboratoire de l'Accélérateur Linéaire d'Orsay. Revue de Physique Appliquée, 1988, 23 (9), pp.1475-1482. 10.1051/rphysap:019880023090147500 . jpa-00245973

\section{HAL Id: jpa-00245973 https://hal.science/jpa-00245973}

Submitted on 1 Jan 1988

HAL is a multi-disciplinary open access archive for the deposit and dissemination of scientific research documents, whether they are published or not. The documents may come from teaching and research institutions in France or abroad, or from public or private research centers.
L'archive ouverte pluridisciplinaire HAL, est destinée au dépôt et à la diffusion de documents scientifiques de niveau recherche, publiés ou non, émanant des établissements d'enseignement et de recherche français ou étrangers, des laboratoires publics ou privés. 


\title{
Développements techniques en matière d'accélérateurs au Laboratoire de l'Accélérateur Linéaire d'Orsay
}

\author{
B. Mouton et Groupe d'Etudes du SERA \\ Laboratoire de l'Accélérateur Linéaire, 91405 Orsay Cedex, France
}

\begin{abstract}
Résumé. - Le LAL a entrepris depuis environ deux ans un programme de recherche et de développement en vue des collisionneurs linéaires. L'objectif est double, d'une part réaliser des gradients accélérateurs plus élevés à partir de structures accélératrices chaudes en bande $\mathrm{S}$, et d'autre part étudier une nouvelle source HF, le Lasertron, ayant des rendements élevés avec une grande puissance crête.
\end{abstract}

Abstract. - A development program on accelerators started two years ago at LAL. This program includes the study of high accelerating gradients from warm travelling wave structures, essentially at $3 \mathrm{GHz}$, and development of a new high peak power, high efficiency RF source, the Lasertron.

\section{Introduction.}

L'objectif visé à moyen terme est la réalisation d'accélérateurs linéaires collisionneurs $\mathrm{e}^{+} \mathrm{e}^{-}$de très grande énergie.

Afin de limiter la taille de telles machines il est nécessaire d'accroître les gradients d'accélération dans les structúres. Actuellement on réalise de façon courante des gradients de l'ordre de $20 \mathrm{MV} / \mathrm{m}$, mais on envisage qu'un tel seuil puisse être porté au niveau de $100 \mathrm{MV} / \mathrm{m}$ soit en améliorant les structures classiques soit en développant de nouvelles structures. Dans ce dernier cas l'accent doit être mis sur l'efficacité des structures, c'est-à-dire la capacité à transformer la puissance $\mathrm{HF}$ injectée en champ accélérateur.

Pour une structure donnée la puissance HF qu'il faut fournir est proportionnelle au carré du gradient accélérateur qu'il faut produire. En clair il faut être capable de fournir les puissances HF correspondantes, et ceci dans les meilleurs conditions de rendements possibles.

Le laboratoire de l'Accélérateur Linéaire s'est engagé depuis quelque temps, parallèlement à d'autres laboratoires dans le monde, à contribuer à ce double objectif qui est d'une part de pousser la technologie des structures accélératrices conventionnelles, ou quasi conventionnelles, et d'autre part de développer des nouvelles sources de puissance HF à très grande puissance crête et à haut rendement.

\section{Etudes forts gradients.}

2.1 Problèmes À RÉSOUdRE. - Les problèmes que l'on rencontre et qui limitent le champ accélérateur sont de trois types, physiques, chimiques et l'interaction onde-faisceau.
2.1.1 Problèmes physiques. - La limitation du champ dépend :

- des dimensions et de la forme des cavités qui interviennent dans la réalisation d'un rapport optimum $E_{\text {acc }} / E_{\text {surface }}$. Il faut rechercher des formes telles que le facteur de qualité $Q$ et le rapport $r / Q$, où $r$ représente l'impédance shunt par unité de longueur, soient le plus grand possible tout en maintenant le rapport $E_{\text {acc }} / E_{\text {surface }}$ compatible avec la limite de claquage.

- de l'auto-émission électronique initiée par l'émission secondaire, l'émission de champ et la thermo-émission.

L'émission d'électrons secondaires due au bombardement ionique entraîne un processus de cascade, qui augmente le courant ainsi émis jusqu'au claquage, processus décrit par la formule de Kilpatrick : (Réf. [1])

$$
f=1,643 E_{\max }^{2} \mathrm{e}^{-8,5 / E_{\max }}
$$

pour une fréquence $f=3 \mathrm{GHz}$ on obtient $E_{\max }=46,8 \mathrm{MV} / \mathrm{m}$

- de la largeur de l'impulsion HF ;

- de la nature du matériau;

- de la fréquence de fonctionnement. Sachant que le seuil de claquage et que $r / Q$ augmentent avec la fréquence on aura intérêt à utiliser des fréquences plus élevées par exemple $6 \mathrm{GHz}$ au lieu de $3 \mathrm{GHz}$.

Un autre problème est celui des puissances mises en jeu. Pour ce faire il faut développer des sources de puissance pulsée avec un meilleur rendement $(\eta \geqslant 75 \%)$, type Lasertron et fonctionnant en impulsions courtes. 
Il faut aussi développer des systèmes de compression de l'impulsion $\mathrm{HF}$ avec un meilleur rendement. Il s'agit d'une forme de stockage, suivie d'une régénération rapide de l'énergie contenue dans une impulsion de klystron. Le stockage s'effectuant dans des cavités à fort $Q$ (très surcouplées) dont le temps de remplissage est inférieur à la durée de l'impulsion du klystron. Une amélioration possible est de faire une adaptation séparée entre le klystron et la cavité d'une part et d'autre part entre la cavité de stockage et la section accélératrice, ce qui conduirait à des puissances crêtes très grandes sur des durées très petites.

\subsubsection{Problèmes chimiques. - Les problèmes que} l'on s'attend à rencontrer relèvent de :

- la chimie des surfaces : recombinaison sur la surface par oxydation, dopage, nettoyage ;

- la métallurgie : types de soudures;

- la chimie des dépôts ;

- la contamination chimique des surfaces.

\subsubsection{Problèmes liés à l'interaction onde-faisceau}

- phénomènes transitoires ;

— instabilité et pertes de faisceau.

\subsection{Programme DE DÉVELOPPEMENT MIS EN} CEUVRE AU LAL.

2.2.1 Type de structure. - A court terme le programme du LAL est de déterminer la limite supérieure opérationnelle du champ accélérateur d'un élément de section accélératrice classique caractérisée par une structure périodique à iris, travaillant en bande $\mathrm{S}(3 \mathrm{GHz})$. La structure en question est du type LIL (Linac Injecteur de LEP) mais plus courte et à impédance constante, c'est-à-dire avec des cellules élémentaires identiques (Figs. 1 et 2).

Les caractéristiques HF de cette structure sont :

- Ondes progressives.

- Bande $S(f=2998,55 \mathrm{MHz}, \lambda=10 \mathrm{~cm})$.

- Mode $2 \pi / 3$ ( 3 cellules par longueur d'ondes).

- Diamètre d'iris $\phi=18 \mathrm{~mm}$.

- Vitesse de groupe : $c / v_{\mathrm{g}}=137$.

- Longueur : $L=0,5 \mathrm{~m}$.

- Facteur de qualité : $Q=15000$.

- Impédance shunt par mètre : $r=74 \mathrm{M} \Omega / \mathrm{m}$.

Les pertes par effet Joule dans les parois conduisent à une décroissance exponentielle du champ électrique axial :

$$
E=E_{0} \mathrm{e}^{\frac{-\omega}{2 v_{\mathrm{g}} Q} z}
$$

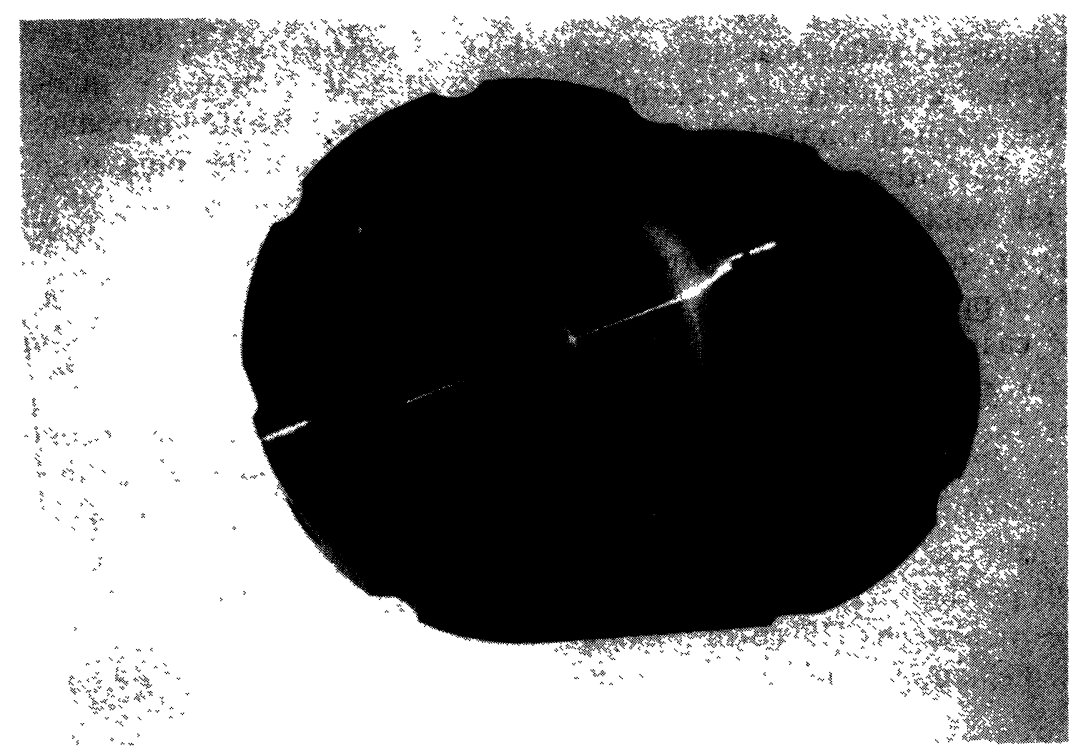

Fig. 1. - Cellule élémentaire.

[Unit Cell.]

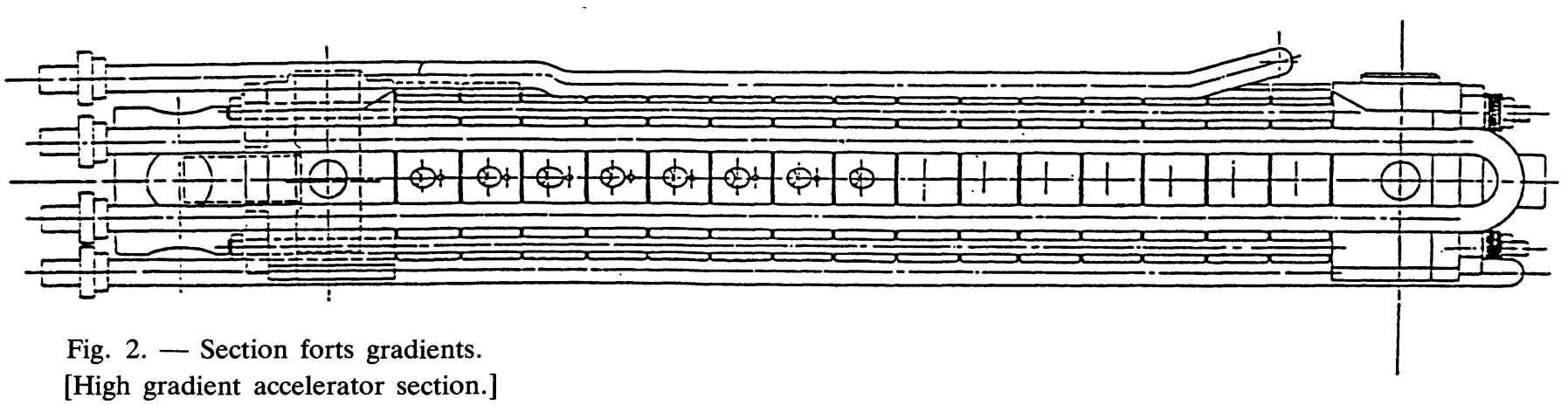

[High gradient accelerator section.] 
En onde progressive le temps de remplissage de la structure est :

$$
T_{\mathrm{r}}=L / v_{\mathrm{g}}
$$

On voit que sur la longueur totale de la structure le champ accélérateur est atténué d'un facteur $\mathrm{e}^{-\tau}$ où le coefficient d'atténuation $\tau$ vaut :

$$
\tau=\frac{\omega}{2 Q} T_{\mathrm{r}}
$$

L'énergie totale acquise par une particule lors de la traversée de la structure peut s'exprimer en fonction de ce coefficient :

$$
V(\tau)=\left(P_{0} r L\right)^{1 / 2}\left[(2 \tau)^{1 / 2} \frac{\left(1-\mathrm{e}^{-\tau}\right)}{\tau}\right] .
$$

Par ailleurs l'efficacité de la structure s'exprime par :

$$
\eta_{\mathrm{s}}=\frac{V^{2}(\tau)}{V^{2}(0)}
$$

où $V(0)$ serait le gain d'énergie en l'absence de pertes.

On a donc :

$$
\eta_{\mathrm{s}}=\left(\frac{1-\mathrm{e}^{-\tau}}{\tau}\right)^{2}
$$

On montre alors que la puissance nécessaire à l'entrée de la section pour obtenir un gain d'énergie donné présente un minimum (plat) pour $\tau=1,26$ ce qui dans la pratique signifie une section relativement longue, et que ce minimum correspond à un rendement relativement faible $\left(\eta_{\mathrm{s}}=32 \%\right)$.

Une augmentation du rendement passe par une diminution du coefficient d'atténuation ce qui suggère à la fois un raccourcissement des sections et une augmentation de la puissance à l'entrée, naturellement suivis d'une augmentation de gradient accélérateur (Réf. [2]).

Ceci est encore plus vrai lorsque l'on utilise la compression d'impulsion $\mathrm{HF}$ à l'entrée de la section (Réf. [3]).

Du point de vue technologique la structure précédemment décrite est faite de cellules en Cuivre (BE58) assemblées à relativement basse température par diffusion d'argent. Cette technologie cependant ne permet pas d'avoir une bonne étanchéité au vide c'est pourquoi la structure est enfermée dans une enveloppe à vide en inox. L'accord de chaque cellule est fait par déformation des parois.

Un deuxième type de structure est actuellement en développement toujours à partir des cellules LIL, mais avec une autre technologie de construction. Cette structure d'une longueur comprise entre $0,9 \mathrm{~m}$ et 1,2 m, sera assemblée par brasure à haute température sous vide, et ne comportera pas d'enveloppe à vide (section auto-étanche). Les coupleurs d'entrée- sortie seront du type LIL et identiques, ce qui détermine l'intervalle de choix du diamètre d'iris des cellules $17,977 \leqslant 2 a \leqslant 18,522 \mathrm{~mm}$.

La structure étant de type à impédance shunt constante, le seul autre paramètre libre est la longueur. Le choix s'est porté sur une section de longueur $1 \mathrm{~m}$, coupleurs non compris, et de diamètre d'iris $18 \mathrm{~mm}$. Dans l'éventualité d'un fonctionnement en mode avec compression d'énergie, de cette structure on peut s'attendre à obtenir un gradient de $100 \mathrm{MV} / \mathrm{m}$ pour une puissance de $33 \mathrm{MW}$.

Un troisième type de structure est en cours de réalisation en collaboration avec la CGR-MeV. C'est une structure à onde progressive inverse, avec couplage magnétique en mode $4 \pi / 5$, l'impédance shunt sera proche de $100 \mathrm{M} \Omega / \mathrm{m}$ et le $c / v_{\mathrm{g}}<50$ donc cette structure aura un faible temps de remplissage. Cette structure aura une longueur d'un mètre, sera assemblée par diffusion d'argent et comportera une enveloppe à vide.

2.2.2 Tests. - Deux étapes sont prévues, une avec la puissance $\mathrm{HF}$ seule, l'autre avec la puissance $\mathrm{HF}$ et un faisceau d'électrons.

Les tests avec puissance HF seule permettront de déterminer les seuils éventuels de claquage dans la limite de la puissance HF disponible, et le taux d'émission de champ.

Les tests avec faisceau, qui nécessitent l'installation d'une station d'essais, permettront de déduire, à partir de l'énergie des électrons accélérés, l'amplitude exacte du champ électrique. Il est également prévu d'étudier l'effet d'un faisceau sur les seuils de claquage éventuels.

En effet un faisceau relativement intense peut induire des champs électromagnétiques sur des modes parasites de la structure et de ce fait modifier les conditions de champ électrique sur les parois.

2.3 STATIONS D'ESSAIS FORT GRADIENT (Fig. 3). Pour mener à bien le programme décrit ci-dessus une station d'essais est en cours d'installation.

2.3.1 Source de puissance. - On utilise un klystron $3 \mathrm{GHz}, 35 \mathrm{MW}$ crête, de durée d'impulsion 4,5 $\mu \mathrm{s}$ associé au modulateur prototype LIL. Trois modes d'utilisation sont possibles.

- Utilisation directe : le klystron débite directement dans le coupleur d'entrée de la section. Pour une puissance de $35 \mathrm{MW}$, on s'attend à avoir un champ accélérateur maximum à l'entrée de la section de $38 \mathrm{MV} / \mathrm{m}$.

- Utilisation avec compression d'impulsion (LIPS = LEP Injector Power Saver). LIPS utilise des cavités de stockage d'énergie à faible perte ( $Q \simeq 180000$ ) et des impulsions longues de klystron $(\simeq 4,5 \mu \mathrm{s})$. Après compression la puissance crête sera de $260 \mathrm{MW}$, ce qui conduira à un champ de l'ordre de $100 \mathrm{MV} / \mathrm{m}$ à l'entrée de la section. 


\section{STATION FORT GRADIENT}

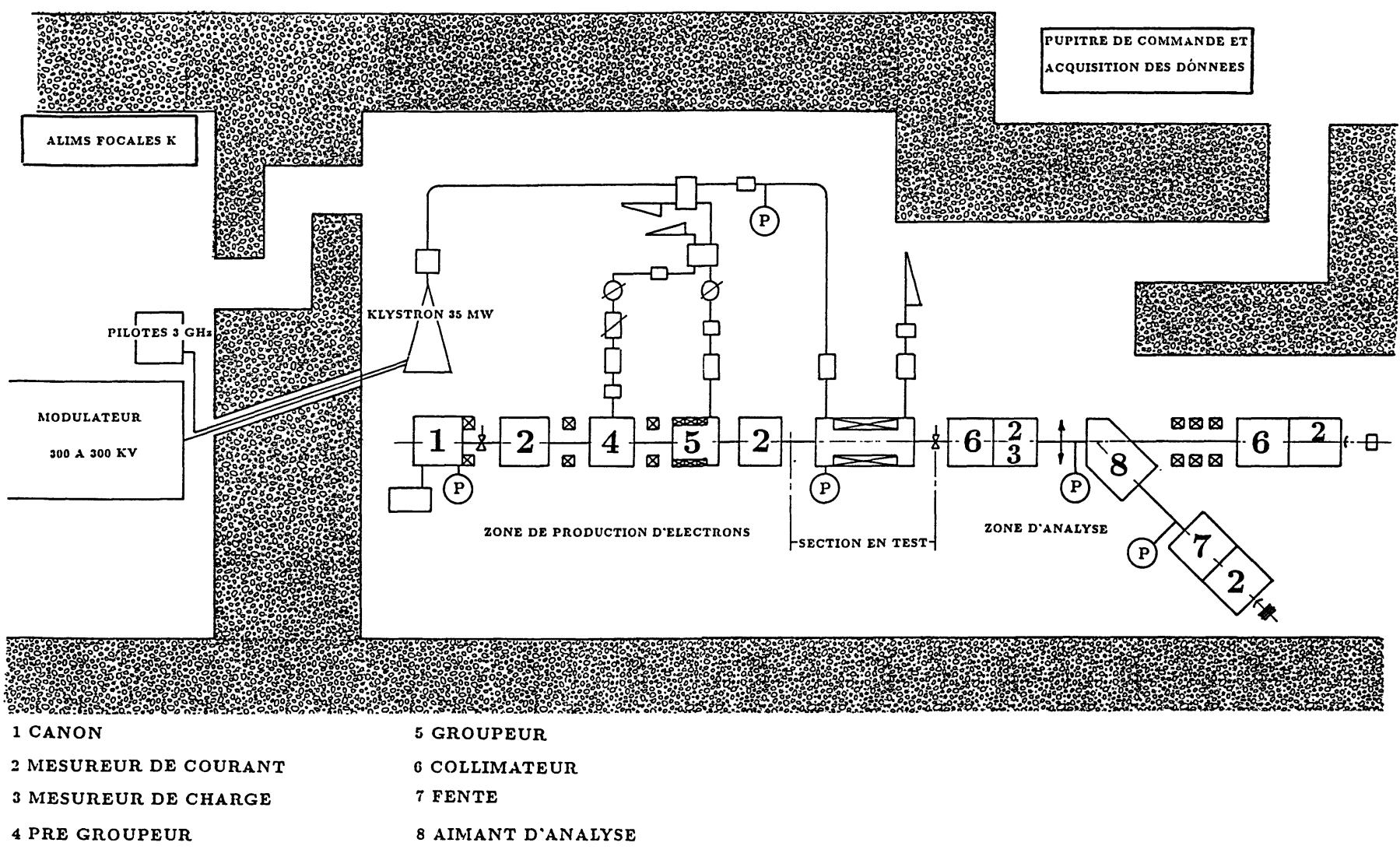

Fig. 3. - Station d'essais forts gradients.

[High gradient test facility.]

- Utilisation de la recirculation de la puissance HF en incluant la section dans un anneau résonnant. La recirculation consiste à réutiliser la puissance non dissipée en sortie de la structure pour alimenter l'entrée de celle-ci, la recombinaison étant effectuée par un coupleur directif. Dans ce cas l'on espère une puissance de $135 \mathrm{MW}$ donnant un champ maximum de $75 \mathrm{MV} / \mathrm{m}$. L'énergie obtenue dans ce cas est plus faible que dans le cas LIPS, mais l'on travaille en impulsions longues ce qui constitue un test « forts gradients » plus probant. Cette solution, permettant d'accélérer des impulsions longues d'électrons, peutêtre intéressante pour certaines applications.

2.3.2 Distribution HF. - La ligne de base est un guide d'onde WR284 à paroi épaisse $(\simeq 4 \mathrm{~mm})$ refroidie, sous vide, à l'exception d'un sas $\mathrm{SF}_{6}$ entre la fenêtre céramique du klystron et une fenêtre d'entrée sur le guide.

2.3.3 Source d'électrons. - Un canon de type SLAC, avec modulateur associé réalisé au LAL, fonctionnant à $70 \mathrm{KV}$ et délivrant des impulsions de courant $0,5<I<1$ A avec une durée $2<\tau<20$ ns servira de source d'électrons.
2.3.4 Lignes de faisceau. - Après le canon on trouve un prégroupeur, un groupeur, un ensemble de mesures (collimateurs/mesureurs), la structure à tester, un deuxième ensemble de mesures puis un aimant d'analyse $45^{\circ}$ de $35 \mathrm{MeV}$ d'induction maximale $1460 \mathrm{G}$.

Sur la voie déviée, une fente d'analyse suivie d'un ensemble de mesures permettra de mesurer l'énergie en valeur absolue et de déterminer le spectre courant-énergie. La voie directe, elle, sera équipée d'un triplet associé à un ensemble de mesures, dans le but de faire une mesure d'émittance par la méthode des trois gradients.

\section{Le lasertron.}

3.1 PRINCIPE (Réf. [4]). - Le lasertron est un tube hyperfréquence dans lequel les électrons produits par photoémission sont déjà groupés au départ de la photocathode dans la mesure où le faisceau laser qui l'illumine est lui-même pulsé (Fig. 4).

Dans l'hypothèse où la photoémission peut être considérée comme quasi instantanée, la qualité du groupement dépend de la qualité du faisceau laser. 


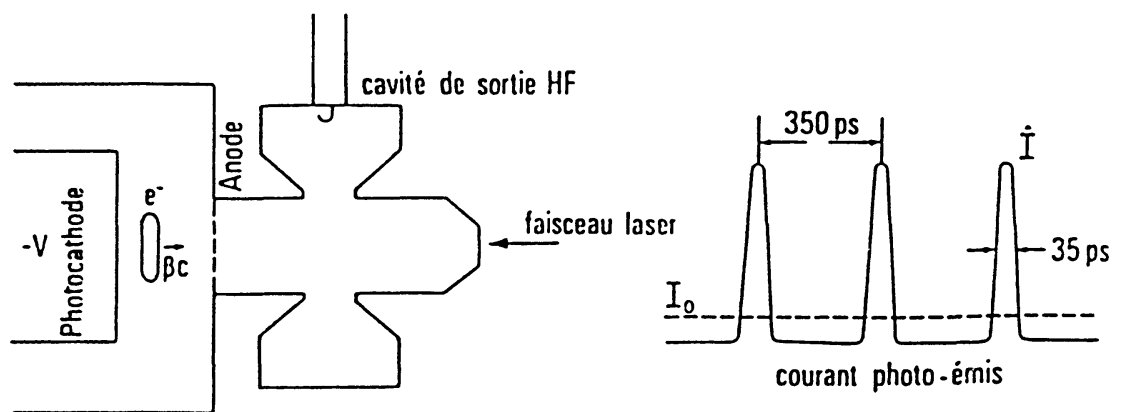

Fig. 4. - Principe du lasertron.

[Schematic diagram of a lasertron.]

Cela n'est en fait que partiellement vrai car pour des densités de courant émis importantes il est nécessaire de considérer les effets répulsifs de la charge d'espace.

Pour maitriser de forts courants il est donc nécessaire d'appliquer une tension d'accélération aussi grande que possible, ce qui en définitive va dans le sens d'une augmentation de la puissance faisceau.

Le laser définit à la fois la fréquence de répétition des impulsions de courant et la longueur totale du train d'impulsions. Il est donc tout à fait possible d'appliquer une tension continue d'accélération.

On comprend mieux l'intérêt du lasertron si on rappelle que dans le cas du klystron le faisceau émi de façon continue doit ensuite être groupé, et que pour qu'un tel groupement soit efficace il faut que les vitesses restent non relativistes ce qui forcément limite la tension utile d'accélération.

Par ailleurs un klystron pulsé nécessite l'application d'une tension d'accélération elle-même pulsée obtenue à partir d'un modulateur de puissance.

Dans les deux cas, klystron et lasertron, la puissance HF est extraite à partir de l'harmonique fondamental du train d'impulsions de courant grâce à une cavité résonnante d'extraction couplée à un guide d'onde.

\subsection{Simulation DES EFFETS DE CHARGE D'ESPACE.}

En régime d'impulsions très brèves, la charge électrique maximum que l'on peut prétendre extraire de la photocathode est la charge superficielle c'est-à-dire :

$$
Q_{\mathrm{m}}=C V=\frac{\varepsilon_{0} S}{d} V
$$

où $C$ est la capacité de l'espace cathode-anode, $V$ la tension appliquée, $S$ la surface de la cathode et de la distance cathode-anode.

Dans le cas d'une impulsion de courant très courte, par rapport à son extension radiale, on peut admettre que le champ électrique de charge d'espace, qui s'oppose au champ électrique d'accélé- ration, est purement azimuthal et donné en première approximation par

$$
E_{\text {c. e. }}=\frac{Q}{2 \varepsilon_{0} S} .
$$

La limite de charge d'espace est alors obtenue lorsque $E_{\text {c. e. }}=E_{\text {acc }}$ d'où :

$$
Q_{1}=2 \varepsilon_{0} S E_{\mathrm{acc}}=\frac{2 \varepsilon_{0} S}{d} V
$$

c'est-à-dire le double de la charge superficielle précédemment définie.

Il ressort de ce modèle simplifié à une dimension que la charge maximum extraite ou « charge critique » $Q_{\text {c }}$ est donnée par la charge superficielle et non pas par l'effet de charge d'espace.

Il s'ensuit une propriété caractéristique du lasertron à savoir que si $f$ est la fréquence de répétition des impulsions on a :

$$
I=f C V
$$

c'est-à-dire que le courant moyen maximum extrait est proportionnel à la tension, alors que dans le cas du klystron la dépendance est en $V^{3 / 2}$.

La dépendance linéaire a été mise en évidence par une équipe japonaise (Réf. [5]) sur un prototype lasertron de basse puissance.

Une fois la charge électrique extraite, et alors que les particules ont encore des vitesses relativement faibles l'effet de charge d'espace, essentiellement électrique, a tendance à ralentir les particules de la queue par rapport aux particules de la tête de l'impulsion créant ainsi une déformation longitudinale du paquet et dégradant le contenu de l'harmonique fondamental du faisceau impulsionnel. Ceci conduira à une perte de rendement au niveau de l'extraction HF.

Le problème est rendu encore plus complexe lorsqu'on considère l'effet de charge d'espace dans la direction tranverse. 
Afin de mieux comprendre ces effets et leur conséquence sur les performances du lasertron un programme de simulation 2-D, de la dynamique des particules dans le laserton a été conçu au LAL.

Ce programme, appelé RING (Réfs. [7] et [8]), permet de traiter des impulsions de faisceau très courtes en régime transitoire depuis son extraction de la cathode jusqu'à l'entrée dans la cavité d'extraction, et en régime permanent pendant la traversée de cette cavité. Il permet l'extraction de paquets selon diverses conditions initiales relatives au laser, puis calcule, ensuite les déformations longitudinales et radiales des micro-paquets dans la traversée de l'espace canon, pour enfin permettre d'optimiser les caractéristiques de la cavité d'extraction HF pour un rendement d'extraction maximum.

On retiendra principalement des simulations effectuées avec RING que la géométrie plane de la cathode est optimale pour le lasertron, que les contraintes de focalisation, de claquage dans les zones $\mathrm{HF}$ et canon, et la sensibilité à la durée de l'impulsion laser augmentent avec la fréquence ( 3 ou $6 \mathrm{GHz})$ du système de manières relativement diverses, la durée de l'impulsion laser ayant un rôle très critique à $6 \mathrm{GHz}$. Le rendement s'avère fortement dépendant de la tension d'accélération du canon à titre d'exemple, un gain de l'ordre de $20 \%$ peut être obtenu en augmentant celle-ci de 300 à $450 \mathrm{kV}$. Nous avons mis aussi en évidence une limite induite par la charge d'espace pour la puissance extractible à la cathode, fonction du champ accélérateur moyen sur la cathode et du temps que va mettre le laser pour extraire la charge maximale, différente de celle rencontrée pour les faisceaux continus.

3.3 ETUDES PROTOTYPES. - Des études particulières de photocathodes en régime impulsionnel fort courant ont été faites au LAL et font l'objet d'une autre contribution du LAL à cette conférence (Réf. [6]).

L'objectif à moyen terme est la réalisation d'une maquette lasertron afin de démontrer la faisabilité d'un tel tube. Cette étape se fera à une puissance comparable à celle des klystrons pulsés conventionnels.

les principaux paramètres retenus sont :

- Fréquence HF : $6 \mathrm{GHz}$.

- Puissance crête : $\simeq 20 \mathrm{MW}$.

- Rendement $: \geqslant 75 \%$.

La fréquence usuelle des accélérateurs linéaires est $3 \mathrm{GHz}$. Cependant il est souhaitable d'aborder les études de futurs collisionneurs, pour des raisons d'efficacité des structures accélératrices, à plus haute fréquence. C'est dans cet esprit que le choix actuel du LAL s'est porté sur $6 \mathrm{GHz}$, conjointement avec le fait qu'il existe d'ores et déjà un klystron à cette fréquence ce qui permettra une comparaison des performances.

Pour l'instant il a été conçu et réalisé une enceinte à ultravide (Fig. 5) capable de tenir une très haute

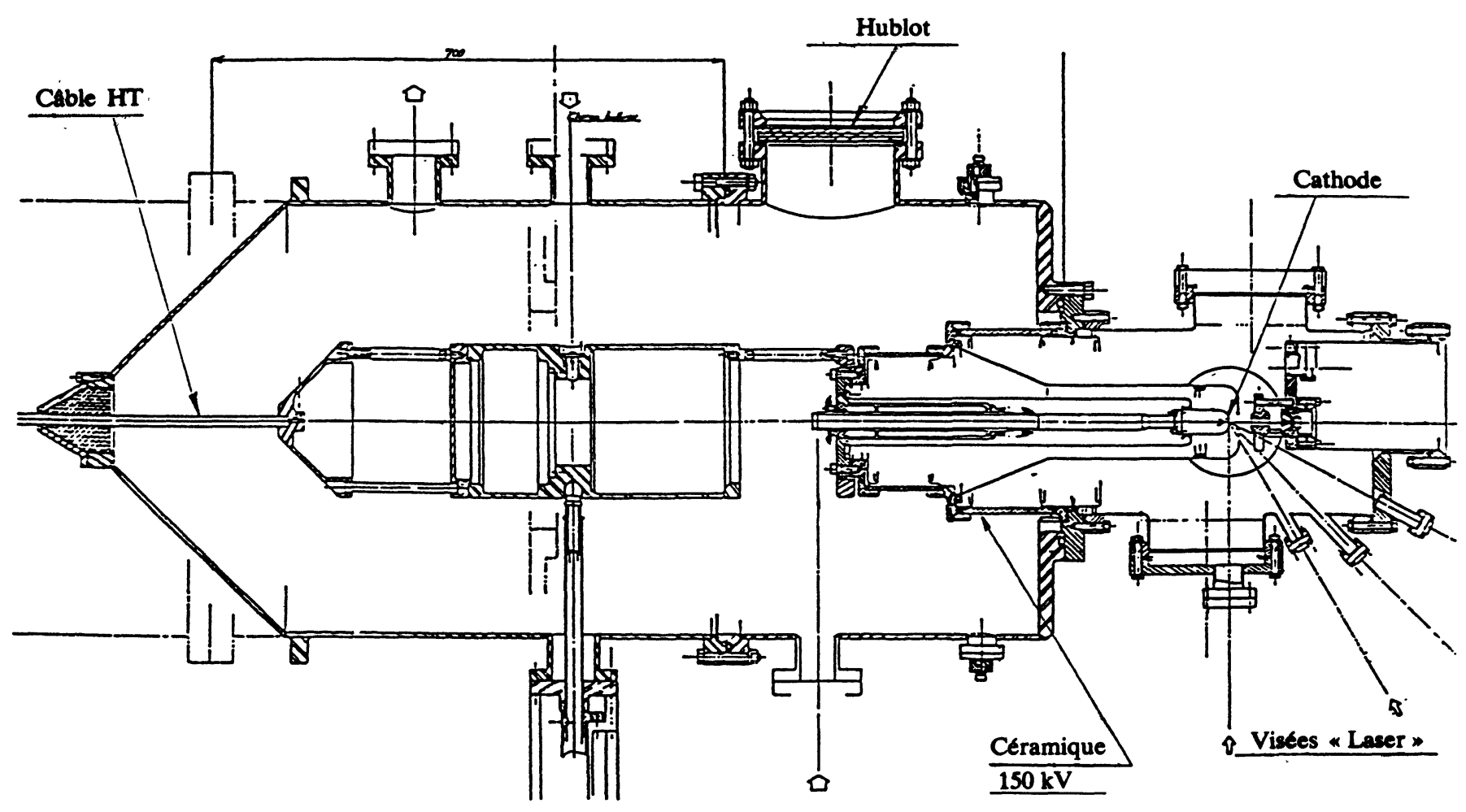

Fig. 5. - Enceinte d'étude des photocathodes.

[Experimental vessel of photocathodes.] 
tension continue, soit $150 \mathrm{kV}$ dans un premier temps et $400 \mathrm{kV}$ plus tard. L'enceinte est destinée d'une part à prolonger les expériences photocathodes en fort courant impulsionnel dans un environnement très haute tension, d'autre part à faire les premiers tests de faisabilité du lasertron.

L'enceinte a été conçue en module si bien que la partie côté anode peut être séparée aisément de la partie HT et ceci afin de faciliter l'interchangeabilité des moyens de diagnostic des faisceaux placés derrière l'anode. Les moyens de diagnostic envisagés sont :

a) Cylindre de Faraday type coaxiale large bande. Permettra une première visualisation des impulsions de courant.

b) Moniteur de rayonnement de transition. Permettra une conversion électron-photon pour analyse du train d'impulsions avec une caméra à fente.

c) Cavité HF de déflection. Permettra une analyse fine de la longueur des impulsions élémentaires (résolution de l'ordre de la picoseconde).

d) Cavité d'extractions HF. La fréquence de résonance de cette cavité correspond à la fréquence finale de modulation des impulsions laser. Ce montage permettra une étude du rendement du lasertron.

Dans la configuration $d$ ) l'enceinte d'étude des photo cathodes devient une véritable maquette lasertron. On se propose donc d'étudier cette maquette de faisabilité en deux temps :

1) $H T=150 \mathrm{kV}$.

Les résultats de la simulation avec le programme RING nous prédisent les performances suivantes :

- Fréquence HF

$6 \mathrm{GHz}$

- Puissance HF

$1,2 \mathrm{MW}$

- Rendement $60 \%$

- Puissance faisceau

$2 \mathrm{MW}$

- Courant moyen

$13,3 \mathrm{~A}$

$2,2 \mathrm{nC}$

$1,1 \mathrm{~cm}^{2}$

- Surface effective de la cathode

- Champ magnétique

$3,5 \mathrm{kG}$

Cette étape nécessite un laser dont les performances minimum sont :

- Fréquence optique

- Fréquence de modulation

- Largeur de l'impulsion élémentaire

- Energie de la micro-impulsion UV $6 \mathrm{GHz}$ $35 \mathrm{ps}$ $<1 \mu \mathrm{J}$
La largeur totale du train d'impulsions devant être si possible $>100 \mathrm{~ns}$ et la fréquence de répétition $\geqslant 10 \mathrm{~Hz}$.

Cette étape permettra de vérifier les prédictions de la simulation et de ce fait préparera à l'étape suivante :

2) $H T=400 \mathrm{kV}$.

Dans ce cas les résultats de la simulation avec le programme RING nous prédisent les performances suivantes :

- Fréquence $\mathrm{HF}$

$6 \mathrm{GHz}$

- Puissance HF

$19 \mathrm{MW}$

- Rendement $75 \%$

- Puissance faisceau $25 \mathrm{MW}$

- Courant moyen $62,5 \mathrm{~A}$

- Charge de la micro-impulsion

$10,4 \mathrm{nC}$

- Surface effective de la cathode

$1,1 \mathrm{~cm}^{2}$

- Champ magnétique

$2,8 \mathrm{kG}$

avec les performances ci-dessous par le laser :

- Fréquence optique

UV

- Fréquence de modulation

$6 \mathrm{GHz}$

- Longueur de l'impulsion élémentaire 35 ps

- Energie de la micro-impulsion

$1 \mu \mathrm{J}$

Le laser doit être capable de produire un long train d'impulsions picosecondes, modulées à la fréquence HF désirée, et ceci avec un minimum de modulation d'amplitude. La fréquence de répétition souhaitée devra être de quelques dizaines de Hertz.

\section{Conclusion.}

Le programme de développement technique démarré au LAL, et qui s'inscrit dans un contexte très large de développement en matière d'accélérateurs futurs, a maintenant dépassé la phase préparatoire pour entrer dans la phase expérimentale proprement dite.

Cette deuxième phase devrait s'étendre sur une période minimum de deux ans pour déboucher ensuite sur de nouvelles décisions concernant la phase d'application.

Toutefois dans la période actuelle où les nouvelles idées sont extrêmement prolifiques il est nécessaire de maintenir un programme de développement suffisamment flexible pour intégrer éventuellement certaines de ces nouvelles idées. 


\section{Bibliographie}

[1] KilpatricK, W. D., Criterion for Vacuum Sparking Designed to Include Both RF and DC UCRL 2321 Sept. 1953.

[2] Wilson, P. B., SLAC PUB 3674 Nov. 1985.

[3] LE DuFF, J., Optimization of TW acceleratiny Structures for SLED type modes of operation LAL/RT/84-01.

[4] LE DuFF, J., Introduction au Lasertron LAL/RT/8504.
[5] YoshiOKA, M., et al., Lasertron : laser triggered RF source for linacs in TEV region; Linac Conférence 1984, p. 469 (Darmstadt, F.R.G.).

[6] Boussoukaya, M., et al., Photoémission en régime impulsionnel sur des microcathodes, LAL/RT/87-08.

[7] Tallerico, P., Coulon, J. P., A Ring model of the Lasertron LAL/RT/87-02.

[8] Dubrovin, A., Coulon, J. P., Simulation des performances du Lasertron S.E.R.A. 87-116. 